\title{
Modelling Of Heat Transfer In Turbulent Bubbly Flows Using Direct Numerical Simulations
}

\author{
${ }^{1}$ Sonolet Aymeric, ${ }^{1}$ Guillaume Bois, ${ }^{2}$ Adrien Toutant \\ ${ }^{1}$ Université Paris-Saclay, \\ CEA, Service de Thermo-hydraulique et de Mécanique des Fluides \\ (STMF), 91191 Gif-sur-Yvette, France \\ aymeric.sonolet@cea.fr; guillaume.bois@cea.fr \\ ${ }^{2}$ PROMES-CNRS (8521), Université de Perpignan \\ Perpignan, France \\ adrien.toutant@univ-perp.fr
}

\section{Extended Abstract}

The study of heat transfer in bubbly flows is of great interest in many industrial applications, and especially the ones using boiler systems (such as solar power plants or nuclear reactors). The CEA aspires to create a numerical nuclear reactor which requires a great knowledge of two phase flows. In order to improve our understanding of accidental scenarios, the study and the modelling of complex dynamics and heat transfer of bubbly flows is necessary.

In order to achieve these objectives, numerical simulations are conducted at different scales. The most precise one is the DNS (Direct Numerical Simulations), and the quicker simulation is at the system scale. In this work an original approach is chosen: the DNS will provide reference data for Euler-Euler models (two-fluid Reynolds Averaged NavierStokes), thus resulting in a relatively quick way to resolve heat transfer in turbulent bubbly flows, with a precision inherited from DNS.

The direct simulation of such turbulent bubbly flows, with discontinuities of properties and interfacial transfers, is itself a great challenge, prior to the Euler-Euler modelling. Once this challenge tackled, a reliable statistical database can be produced from the numerical experiments of swarms and bubbly channel flows. So far, as a first step towards more complex boiling flows, simulations are performed without phase change nor mass transfer. As there is no influence of the temperature on the momentum field (passive scalar), multiple thermal fields can be computed using the same momentum.

The front-tracking method is used to manage the bubbles' interfaces. This method was chosen because of its great performance with multi-phase flows, and of the good results obtained in modelling the forces in such flows [1]. The scalar fields are represented on a structured and uniform Eulerian mesh (hexaedric cells, MAC scheme). The one-fluid formulation [2] is used to solve the temperature field with some adjustments made at the interface to tackle issues in mixed cells.

The simulations considered target bubble swarms and turbulent bubbly channel flows. This presentation demonstrates the performance of this algorithm in comparison to other DNS benchmark in the literature: Panda [3] (pure diffusion around a spherical inclusion), Kawamura [4] (single-phase channel flow) and Dabiri and Tryggvason [5] (bubbly channel flow). Then, original results in bubble swarm simulations are presented for various thermal properties. The issue of producing high-quality and high-order statistics in bubble swarms is addressed. Actually, a perfect steady-state cannot be achieved as the temperature difference between phases continuously decreases in time due to the interfacial heat transfer. Three time phases can be distinguished : in the first period wake are not interacting, then the interactions starts and lastly the temperature difference becomes not significant. Finally, the measurement and analysis of heat transfer between the bubbles and the fluid in turbulent conditions are discussed.

In a future work, the issue of thermal modelling will be addressed. the database will be progressively increased by the study of the influence of the bubble Reynolds number, bubbles' deformation, and thermal parameters. 


\section{References}

[1] A. Du Cluzeau, G. Bois, A. Toutant. Analysis and modelling of Reynolds stresses in turbulent bubbly up-flows from direct numerical simulations. Journal of Fluid Mechanics, Cambridge University Press (CUP), 2019, 866, pp.132-168

[2] I. Kataoka, "Local instant formulation of two-phase flow", in International Journal of Multiphase Flow, vol.12, issue 5, 1986, pp. 745-758

[3] A. Panda, Y. Weitkamp, A. Rajkotwala, E. A. J. F. Peters, M. Baltussen, et H. Kuipers, "Influence of Gas Fraction on Wall-to-Liquid Heat Transfer in Dense Bubbly Flows", Chemical Engineering Science: X, sept. 2019, vol. 4, p. 100037

[4] H. Kawamura, H. Abe, et Y. Matsuo, "DNS of turbulent heat transfer in channel flow with respect to Reynolds and Prandtl number effects", in International Journal of Heat and Fluid Flow, vol. 20, issue 3, 1999, pp 196-207

[5] S. Dabiri et G. Tryggvason, "Heat transfer in turbulent bubbly flow in vertical channels", in Chemical Engineering Science, janv. 2015, vol. 122, pp. 106-113 